\title{
LA-UR- $02-7370$
}

Approved for public release;

distribution is unlimited.

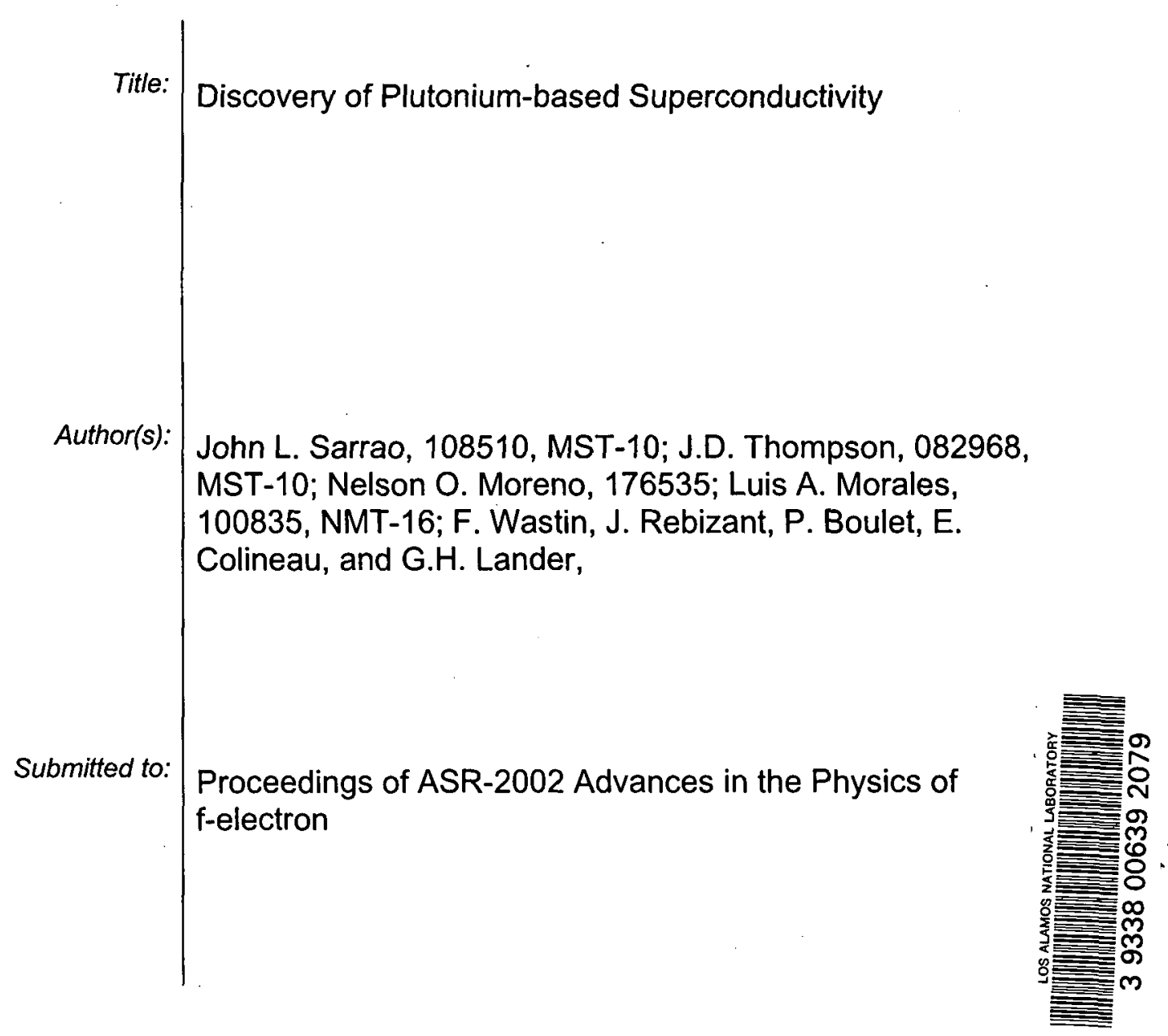

\section{Los Alamos}

NATIONAL LABORATORY

Los Alamos National Laboratory, an affirmative action/equal opportunity employer, is operated by the University of California for the U.S. Department of Energy under contract W-7405-ENG-36. By acceptance of this article, the publisher recognizes that the U.S. Government retains a nonexclusive, royalty-free license to publish or reproduce the published form of this $c$ Ation, or to allow others to do so, for U.S. Government purposes. Los Alamos National Laboratory requests that the publisher identify this - le as work performed under the auspices of the U.S. Department of Energy. Los Alamos National Laboratory strongly supports academic freedom and a researcher's right to publish; as an institution, however, the Laboratory does not endorse the viewpoint of a publication or guarantee its technical correctness. 


\title{
Discovery of Plutonium-Based Superconductivity
}

\author{
J L Sarrao†, J D Thompson†, N O Morenot, L A Morales†, F \\ Wastin $\ddagger$, J Rebizant $\ddagger$, P Boulet $\ddagger$ E Colineau $\neq$, and G H \\ Lander $\dagger$ \\ † Los Alamos National Laboratory, Los Alamos, NM 87545, USA \\ $\ddagger$ European Commission, JRC, Institute for Transuranium Elements, Postfach 2340, \\ 76125 Karlsruhe, Germany

\begin{abstract}
The discovery of superconductivity in single crystals of $\mathrm{PuCoGa}_{5}$ with such a scenario the properties of $\mathrm{PuCoGa}_{5}$ would be intermediate between those of
\end{abstract} \\ transition temperature $\mathrm{T}_{c}=18.5 \mathrm{~K}$ is discussed. The existing data lead to the \\ speculation that the superconductivity in $\mathrm{PuCoGa}_{5}$ may be unconventional. In \\ isostructural $\mathrm{UCoGa}_{5}$ and $\mathrm{CeCoIn}_{5}$, more heavily studied f-electron materials.
}

\section{Introduction}

Plutonium is a fascinating metal whose 5 f electrons are poised on the boundary between localized and itinerant behavior. This instability gives rise to an extremely complex metallurgy[1] and challenges the state of the art in electronic structure calculations[2]. The crossover from localized to itinerant f-electron behavior is also central to the phenomenology of heavy fermion compounds[3].

Here, we discuss a recently discovered microcosm of the fascinating properties of plutonium: the discovery of superconductivity in $\mathrm{PuCoGa}_{5}$ at $18.5 \mathrm{~K}[4]$. Not only is this a rather high $T_{c}$ for an intermetallic compound but also there is at least the suggestion that this superconductivity may be unconventional and, perhaps, spinfluctuation mediated[5, 6].

\section{Evidence for superconductivity in $\mathrm{PuCoGa}_{5}$}

Large single crystals of $\mathrm{PuCoGa}_{5}$ have been grown from an excess $\mathrm{Ga}$ flux. Further and independently, single-crystal platelets have been obtained by arc-melting and subsequent annealing. Physical properties of these materials are identical and reveal bulk superconductivity near $18.5 \mathrm{~K}[4]$. In both cases, single-crystal structural determinations have been made. One finds that $\mathrm{PuCoGa}_{5}$ crystallizes in the $\mathrm{HoCoGa}_{5}$ crystal structure with tetragonal lattice constants $a=4.232 \AA$ and $c=6.786 \AA$. This is the same crystal structure in which $\mathrm{CeMIn}_{5}(\mathrm{M}=\mathrm{Co}, \mathrm{Rh}, \mathrm{Ir})$, a family of unconventional heavy fermion superconductors[7], and $\mathrm{UMGa}_{5}[8]$ also crystallize. 


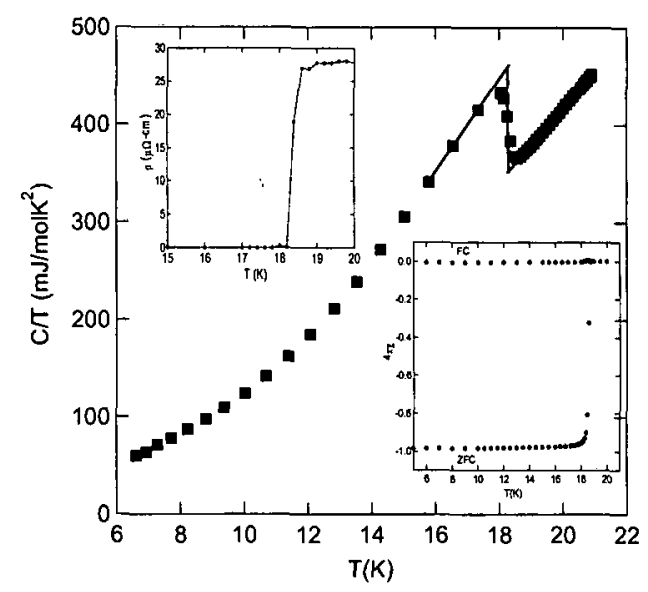

Figure 1. Evidence for superconductivity in $\mathrm{PuCoGa}_{5}$. The main body of the figure shows heat capacity plotted as $\mathrm{C} / \mathrm{T}$ versus $\mathrm{T}$. The upper and lower insets show electrical resistivity and magnetic susceptibility, respectively, as a function of temperature. In all cases signatures of a phase transition are observed in the vicinity of $18.5 \mathrm{~K}$.

In Figure 1 we show the evidence for bulk superconductivity in PuCoGa . A transition to zero resistance, coincident with full-shielding diamagnetism, is observed near $18.5 \mathrm{~K}$. At this same temperature, a step-like transition in heat capacity is observed. If one assumes the $\mathrm{BCS}$ value of $\Delta \mathrm{C} / \gamma \mathrm{T}_{c}=1.43$, then one infers from these data that $\gamma$, a measure of the conduction electron contribution to the low-temperature heat capacity, is $77 \mathrm{~mJ} / \mathrm{molK}^{2}$. This value of $\gamma$ is enhanced relative to that expected for normal metals and is suggestive of heavy fermion behavior.

Interestingly, the $\mathrm{T}_{c}$ of $\mathrm{PuCoGa}_{5}$ decreases from its initial value of $\sim 18.5 \mathrm{~K}$ as a function of time at a rate of approximately $0.2 \mathrm{~K} /$ month. This decrease would seem to be a result of radiation-induced self-damage associated with the spontaneous decay of ${ }^{239} \mathrm{Pu}$. This mechanism is further indicated by the fact that the critical current, $J_{c}$, actually increases with time over the same period[4].

A correspondingly large value of the upper critical field $\mathrm{H}_{c 2}$ in $\mathrm{PuCoGa}_{5}$ has been inferred[4]. In particular, field-dependent resistivity data yield an initial slope of $\mathrm{dH}_{c 2} / \mathrm{dT}$ of $-59 \mathrm{kOe} / \mathrm{K}$. From this value, one can estimate an upper critical field of $740 \mathrm{kOe}$. Further, one can estimate the BCS coherence length and therefore the Fermi velocity, and find that $\gamma \sim 60 \mathrm{~mJ} / \mathrm{molK}^{2}$ in the free-electron limit. Similarly, 


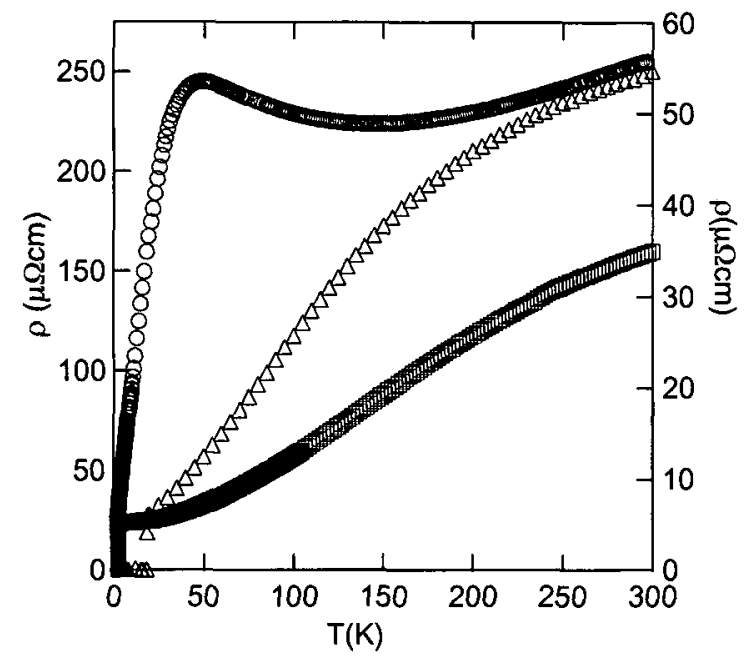

Figure 2. Resistivity as a function of temperature for $\mathrm{CeCoIn}_{5}$ (circles), $\mathrm{PuCoGa}_{5}$ (triangles), and $\mathrm{UCoGa}_{5}$ (squares). The data for $\mathrm{CeCoIn}_{5}$ are plotted on the right axis, whereas $\mathrm{PuCoGa}_{5}$ and $\mathrm{UCoGa}_{5}$ use the left.

from estimates of the thermodynamic critical field, one can estimate $\gamma \sim 70 \mathrm{~mJ} / \mathrm{molK}^{2}$, assuming the BCS value for the condensation energy. Thus, one has several independent estimates of $\gamma \sim 100 \mathrm{~mJ} / \mathrm{molK}^{2}$ in $\mathrm{PuCoGa}_{5}$. Although this is a rather small value compared to other heavy fermion superconductors, it is significantly enhanced compared to $\mathrm{UMGa}_{5}[8]$, in which no superconductivity is observed.

\section{Trends in normal state properties}

Normal-state properties provide further evidence that $\mathrm{PuCoGa}_{5}$ displays stronger electron correlation effects than $\mathrm{UCoGa}_{5}$. Figure 2 displays electrical resistivity for $\mathrm{CeCoIn}_{5}, \mathrm{PuCoGa}_{5}$, and $\mathrm{UCoGa}_{5}$. Judging from the characteristic change in curvature of the temperature dependence of the resistivity, one can see in Figure 2 that this temperature scale is higher in $\mathrm{PuCoGa}_{5}$ than in $\mathrm{CeCoIn}_{5}$, but not as high as in $\mathrm{UCoGa}_{5}$. This trend can be confirmed from heat capacity measurements in these compounds, which find that the linear-in-temperature coefficient of the low-temperature heat capacity, $\gamma$, increases from $\sim 10 \mathrm{~mJ} / \mathrm{molK}^{2}$ in $\mathrm{UCoGa}_{5}$ to $\sim 100 \mathrm{~mJ} / \mathrm{molK}^{2}$ in $\mathrm{PuCoGa}_{5}$ to $\sim 1000 \mathrm{~mJ} / \mathrm{molK}^{2}$ in $\mathrm{CeCoIn}_{5}[7]$.

From these data one is led to the conclusion that the superconductivity in $\mathrm{PuCoGa}_{5}$ may be unconventional. In such a scenario, the order-of-magnitude higher $\mathrm{T}_{c}$ in $\mathrm{PuCoGa}_{5}$ compared to $\mathrm{CeCoIn}_{5}\left(\mathrm{~T}_{c}=2.3 \mathrm{~K}\right)[7]$ would be expected from the increase in bandwidth in going from $5 f$ electrons to 4 f electrons $[9,10]$. It is generally understood that $4 \mathrm{f}$ electrons have a greater degree of localization than do $5 \mathrm{f}$ electrons, as deduced, for example, by the evolution of the Wigner-Seitz radius as a function of atom across the lanthanide/actinide families[11]. 
Although the suggestion of unconventional superconductivity in $\mathrm{PuCoGa}_{5}$ may seem implausible, the alternative, an 18-K conventional, phonon-mediated superconductor, is equally challenging. In $\mathrm{PuCoGa}_{5}$, magnetic susceptibility measurements reveal Curie-Weiss behavior consistent with a paramagnetic moment of $\sim 0.7 \mu_{B} / \mathrm{Pu}[4]$. The pair-breaking tendency of magnetic moments would suggest that $\mathrm{UCoGa}_{5}$, a temperature-independent paramagnet, would have a higher $\mathrm{T}_{c}$ than $\mathrm{PuCoGa}_{5}$, in contrast to what is observed.

\section{Summary}

We have discussed the observation of superconductivity above $18 \mathrm{~K}$ in $\mathrm{PuCoGa}_{5}$. The speculation that this might be unconventional, spin-fluctuation-mediated superconductivity raises the possibility that $\mathrm{PuCoGa}_{5}$ could be an intellectual bridge between the known heavy-fermion superconductors (with characteristic $\mathrm{T}_{c} \sim 1 \mathrm{~K}$ ) and the high- $T_{c}$ cuprates (with characteristic $T_{c} \sim 100 \mathrm{~K}$ ). Thus, the transuranics may represent a particularly fertile, if unplowed, field for the discovery of additional superconductors.

We thank Z. Fisk for fruitful discussions. Work at Los Alamos was performed under the auspices of the U. S. Department of Energy.

\section{References}

[1] Hecker S S 2001 MRS Bulletin 26 672-678.

[2] Savrasov S Y, Kotliar G and Abrahams E 2001 Nature 410 793-795

[3] Smith J L, Fisk Z and Hecker S S 1985 Physica B \& C 130 151-158

[4] Sarrao J L, Morales L A, Thompson J D, Scott B L, Stewart G R, Wastin F, Rebizant J, Boulet P, Colineau E and Lander G H 2002 Nature 420297

[5] Ophale I and Oppeneer P M 2002 preprint

[6] Hotta T and Ueda K 2002 preprint

[7] Petrovic C, Pagliuso P G, Hundley M F, Movshovich R., Sarrao J L, Thompson J D, Fisk Z and Monthoux P 2001 J. Phys. Condens. Matter 13 L337

[8] Grin Yu, Rogl P and Hiebl K 1986 J Less Common Met. 121 497-505

[9] Mathur N D, Grosche F M, Julian S R, Walker I R, Freye D M, Haselwimmer R. K W and Lonzarich G G 1998 Nature 39439

[10] Monthoux P and Lonzarich G G 2001 cond-mat/0207556, and Monthoux P 2002 these proceedings

[11] Wills J M and Eriksson O 2002 Los Alamos Science 26128 
This form is to be completed and submitted to S-7, Classification Group, with copies as required of abstract or paper BEFORE you present or submit for publication any technical work of the Laboratory.

Note: Form will be returned if all sections are not filled out correctly.

Allow three days for S-7 review.

1. Author(s) [full name, (first, middle, last) $Z$ number, and group affiliation]

John L. Sarrao, 108510, MST-10; J.D. Thompson, 082968, MST-10; Nelson O. Moreno, 176535; Luis A. Morales, 100835, NMT-16; F. Wastin, J. Rebizant, P. Boulet, E. Colineau, and G.H. Lander,

\section{Required Copies}

Cover sheet is required on all papers

(Form 836 for LA-UR, Form 1756a-e for LA-CP)

One of this form

Two of unclassified submittal (abstract/vugraphs/paper) One of classified or controlled submittal (abstract) vugraps/paper)

LA-UR/LAP $02-7370$

2. Auth $\phi r(s)$ Signature and Date (optional for S-7)

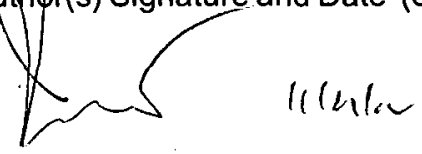

\section{Title of Article (in caps: spell out all symbols)}

Discovery of Plutonium-Based Superconductivity

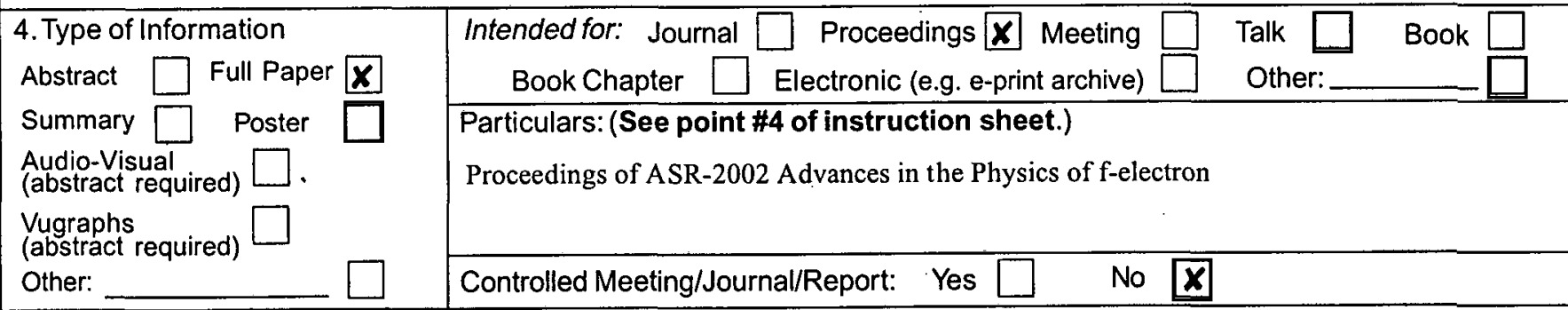

5. List the LA numbers of previous reports that might be useful to the reviewer

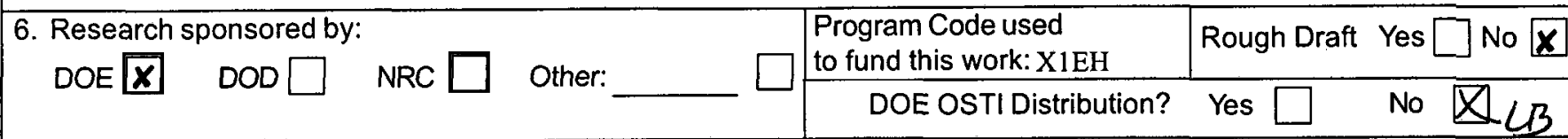

\begin{tabular}{|l|l|l|l|l|}
\hline 7. Funding agency release required: Yes $\square$ No $\square$ Copy Attached: $\quad$ Yes $\square$ & $\square$ \\
\hline $\begin{array}{l}\text { 8. Deadline Date } \\
11 / 26 / 02\end{array}$ & Telephone number and e-mail address for notification of release & Mail Stop \\
K764
\end{tabular}
9. Derivative Classifier Signature or DUSA Designator
Date $11 / 2 / 1 \mathrm{c}$

Classified $\square$ Unćlassified $\triangle 1$ Unclassified, limited

10. Typed/Printed Name of Responsible Party
John L. Sarrao

Signature and $\$$ ate (Reguired)

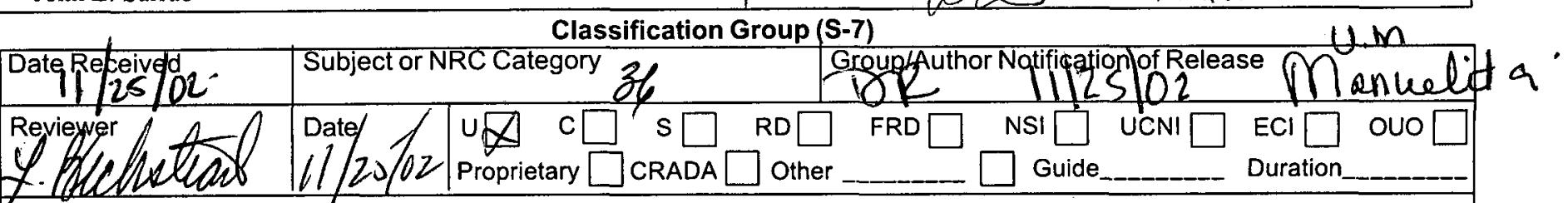

Comments:

Business and Patent Law

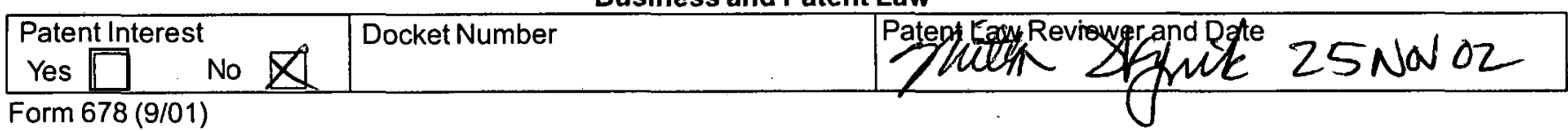

